\title{
The effect of anode surface area on nanoporous oxide formation during anodizing of low purity aluminum (AA1050 alloy)
}

\author{
Leszek Zaraska • Elżbieta Kurowska • \\ Grzegorz D. Sulka $\cdot$ Ilona Senyk • Marian Jaskula
}

Received: 3 June 2013 / Revised: 2 August 2013 / Accepted: 5 August 2013 / Published online: 17 August 2013

(C) The Author(s) 2013. This article is published with open access at Springerlink.com

\begin{abstract}
Porous anodic alumina layers were obtained by a simple two-step anodization of low purity aluminum $(99.5 \%$ Al, AA1050 alloy) in a $0.3 \mathrm{M}$ oxalic acid electrolyte at $45 \mathrm{~V}$ and $20{ }^{\circ} \mathrm{C}$. The effect of anode surface area on structural features of nanoporous oxide and process of oxide formation was investigated. An ordered structure composed of nanostripes or nanopores was formed on the Al surface during electrochemical polishing in a mixture of perchloric acid and ethanol. This nanopattern is then replicated during the anodic oxide formation. It was found that the pore diameter, interpore distance, and porosity increase slightly with increasing surface area of the aluminum sample exposed to the anodizing electrolyte. On the other hand, a slight decrease in pore density and cell wall thickness was observed with increasing surface area of the sample. The detailed inspection of current density vs. time curves was also performed. The obtained results revealed that the higher surface area of the anode, the local current density minimum, was reached faster during first step of anodization and the increase in current density corresponding to the pore rearrangement process was observed earlier. Finally, a dense array of Pd nanowires ( $\sim 90 \mathrm{~nm}$ in diameter) was synthesized by simple electrodeposition of metal inside the channels of through-hole nanoporous anodic alumina templates with relatively large surface areas $\left(4 \mathrm{~cm}^{2}\right)$.
\end{abstract}

Keywords Anodization $\cdot$ Aluminum alloy $\cdot$ Porous alumina . Pd nanowires

\footnotetext{
L. Zaraska $(\bowtie) \cdot$ E. Kurowska $\cdot$ G. D. Sulka $\cdot$ M. Jaskula Department of Physical Chemistry and Electrochemistry, Faculty of Chemistry Jagiellonian University, Ingardena 3, 30-060 Krakow, Poland

e-mail: zaraska@chemia.uj.edu.pl

I. Senyk

Department of Electrochemical Power Engineering and Chemistry, Kiev National University of Technologies \& Design, Kiev, Ukraine
}

\section{Introduction}

During the recent years, fabrication of 1D nanostructures such as nanowires and nanotubes has become a subject of great scientific interest due to extremely promising properties of these materials and their potential applications in many different areas [1]. A considerable attention has been also paid to development a simple and inexpensive method of nanofabrication that can be used not only in a laboratory scale but also could have some potential for a commercial-scale implementation [2]. Among numerous strategies reported in the literature, the use of porous anodic aluminum oxide (AAO) seems to be one of the most popular and easiest ways for a template-assisted synthesis of metallic, semiconductor, oxide, or even polymer nanowire arrays [3]. Nanoporous alumina templates can be synthesized by a simple and cost-effective two-step anodic oxidation of aluminum in acidic electrolytes [4]. This process, which does not require any sophisticated and expensive equipment, results in formation of aluminum oxide layers with a regular, dense structure of hexagonally arranged nanopores. Moreover, structural features of nanoporous alumina membranes such as pore diameter, pore-to-pore distance, thickness of the oxide layer, etc. can be controlled by adjusting anodizing conditions, especially type of electrolyte and applied potential of electrolysis [4]. Some detailed information on the fabrication of anodic alumina membranes and influence of anodizing conditions on structural features and nanopore arrangement of anodic alumina can be found in our previous works [5-8].

Recently, some attention has been also put on the use of low purity aluminum alloys with the $\mathrm{Al}$ content of about $99.5 \%$ (e.g., AA1050 alloy) as a starting material for anodization [9-19]. This kind of substrate is very interesting from an economical point of view due to its reasonable price and availability. On the other hand, fabrication of anodic alumina membranes with a high surface area (up to several square centimeters) can be a key factor in the template-assisted synthesis of nanowires in a large scale. 
The main aim of this work was to find out if there is any correlation between the anode surface area and structural features and nanopore arrangement of anodic alumina layers formed by two-step anodizing of low purity aluminum in an oxalic acid electrolyte. Each step of nanoporous oxide formation (from $\mathrm{Al}$ electropolishing to second anodizing) was discussed in detail. Moreover, we demonstrated a successful fabrication of Pd nanowire arrays using nanoporous anodic alumina membranes with relatively large surface areas.

\section{Experimental}

Synthesis of nanoporous alumina layers

Nanoporous alumina layers were fabricated by two-step anodization of the commercially available aluminum foil (99.5\%, type AA1050). Before anodizing, all samples were degreased in ethanol and acetone. Then, electrochemical polishing was performed in a mixture of perchloric acid (60 wt.\%) and ethanol (1:4 vol.) at $20 \mathrm{~V}$ for $1 \mathrm{~min}$. Pt grid was used as a cathode during electropolishing. After that, the working surface areas of all samples were precisely defined by using an acid-resistant paint. In order to study the influence of surface area on structural features of alumina layers, samples with four different surface areas were used (see Table 1).

All samples were anodized under constant potential of $45 \mathrm{~V}$ for $1 \mathrm{~h}$ at $20^{\circ} \mathrm{C}$ in a $0.3 \mathrm{M}$ oxalic acid solution. Anodic oxide layers were then removed by chemical etching in a mixture of 6 wt. $\% \mathrm{H}_{3} \mathrm{PO}_{4}$ and 1.8 wt. $\% \mathrm{H}_{2} \mathrm{CrO}_{4}$ at $60{ }^{\circ} \mathrm{C}$ for $2 \mathrm{~h}$. As-prepared samples were re-anodized at the same experimental conditions (anodizing potential, electrolyte, and temperature) and the duration of the process was $4 \mathrm{~h}$. After anodizing, samples were rinsed with deionized water and dried. All anodizations were carried out in thermostated double-walled electrochemical cells. Pb plates served as cathodes and aluminum samples as anodes. The distance between both electrodes was about $2 \mathrm{~cm}$.

Fabrication of free-standing alumina membranes with a high-surface area

The remaining $\mathrm{Al}$ substrate was removed in a saturated solution of $\mathrm{CuSO}_{4}$ containing 4 wt. $\%$ of $\mathrm{HCl}$. After that, the

Table 1 Dimensions of samples used for anodization

\begin{tabular}{lll}
\hline Sample type & Size & Surface area $\left(\mathrm{cm}^{2}\right)$ \\
\hline A & $0.5 \times 0.5 \mathrm{~cm}$ & 0.25 \\
B & $1.0 \times 1.0 \mathrm{~cm}$ & 1.00 \\
C & $1.5 \times 1.5 \mathrm{~cm}$ & 2.25 \\
D & $2.0 \times 2.0 \mathrm{~cm}$ & 4.00 \\
\hline
\end{tabular}

chemical etching of the barrier layer from the bottom side of the membrane was performed by immersing the membrane in a 5 wt. $\% \mathrm{H}_{3} \mathrm{PO}_{4}$ solution at $45{ }^{\circ} \mathrm{C}$ for 15 min.

\section{Synthesis of Pd nanowires}

Pd nanowires were synthesized via DC galvanostatic electrodeposition inside the nanochannels of AAO templates. All experiments were carried out in a typical three-electrode cell powered by a Reference 3000 potentiostat (Gamry Instruments). Before electrodeposition, a thin Au layer was sputtered on the bottom side of AAO templates to ensure good electric contact. The prepared membranes served as working electrodes and Pt plates were used as both reference and counter electrodes. Electrodepositions were performed under a galvanostatic regime at the constant current density of $2 \mathrm{~mA} / \mathrm{cm}^{2}$ for $60 \mathrm{~min}$. Solution of $\mathrm{Pd}\left(\mathrm{NO}_{3}\right)_{2}(1 \mathrm{mg} / \mathrm{l})$ in $0.5 \mathrm{M} \mathrm{HNO}_{3}$ was used as an electrolyte. After electrodepositions, samples were rinsed with water and ethanol and remaining $\mathrm{AAO}$ templates were removed by chemical etching in a $1 \mathrm{M} \mathrm{NaOH}$ solution for $60 \mathrm{~min}$. Finally, the samples were carefully rinsed with deionized water then with ethanol and dried.

\section{Characterization of samples}

Structural features of anodic alumina layers and nanowire arrays were examined with a field-emission scanning electron microscope (FE-SEM/Energy-dispersive X-ray spectroscopy (EDS), Hitachi S-4700 with a Noran System 7). The composition of nanowires was confirmed by EDS analyses. Characteristic parameters of AAO membranes were determined from SEM images by using the scanning probe image processor WSxM v. 12.0 [20] and ImageJ software [21] combined with the dedicated computational experiment and corresponding executable publication $[22,23]$ powered by the GridSpace2 platform and Collage Authoring Environment [24].

\section{Results and discussion}

SEM images of aluminum surface before and after electropolishing are shown in Fig. 1a,b (low magnification) and Fig. 1c (high magnification), respectively. It is clearly visible that after electropolishing, Al has an almost flat surface (Fig. 1b) with some pits and bumps which could be attributed to the local allocation of impurities [17]. As can be seen in the high magnification image (Fig. 1c), an ordered structure is composed of nanostripes or nanopores formed during electrochemical polishing. The formation of this kind of nanopatterns, reported and characterized previously for high purity aluminum [25-33], is probably a result of the preferential adsorption of alcohol molecules on surface ridges [28, 34]. The local crosssection profile along the line in Fig. 1c (Fig. 1d) indicates that 
Fig. 1 SEM images of aluminum surface before (a) and after electropolishing $(\mathbf{b}, \mathbf{c})$ together with an intensity profile along the blue line in $\mathbf{c}(\mathbf{d})$. The surface area of the sample was $0.25 \mathrm{~cm}^{2}$
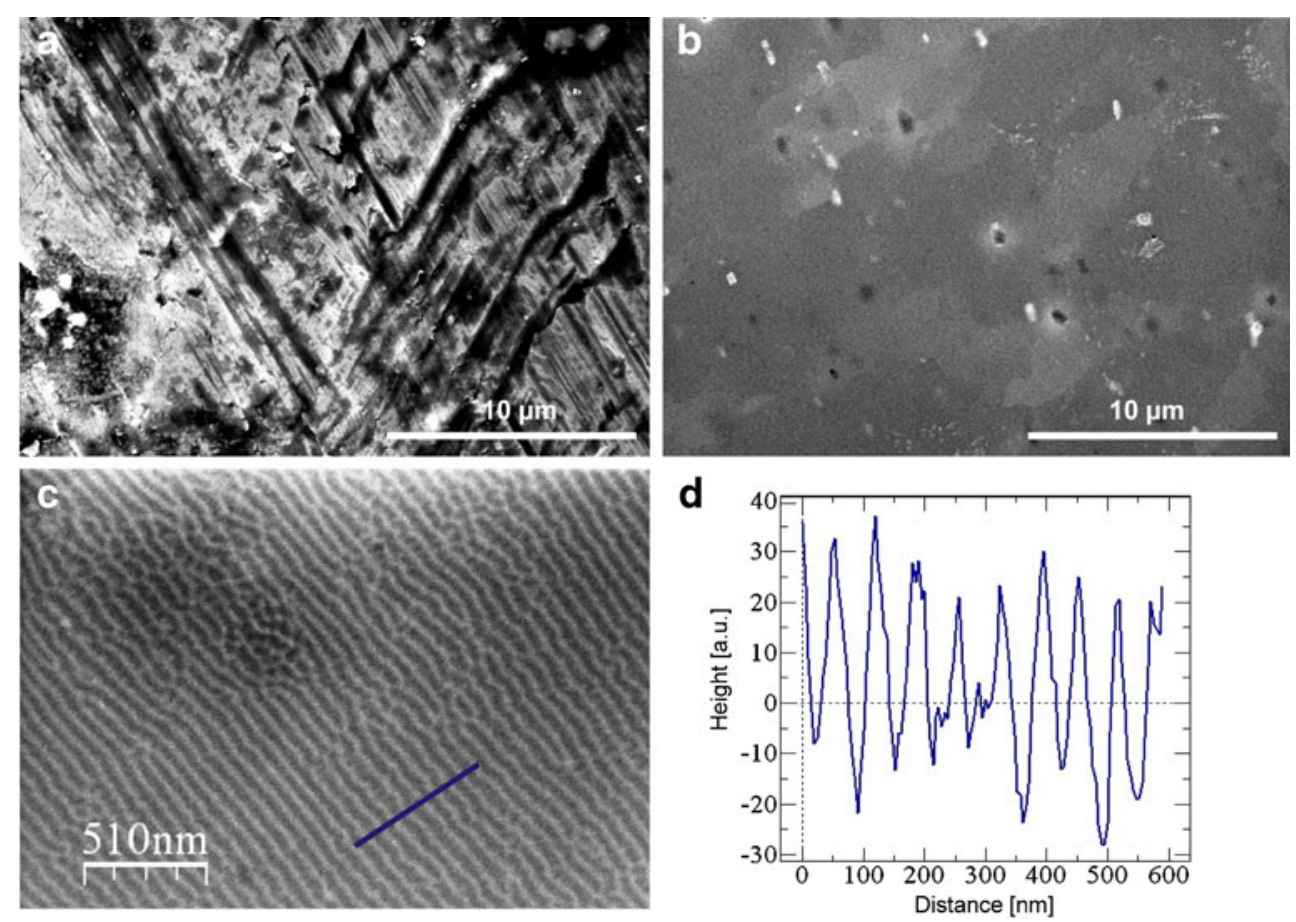

the wavelength of the created stripe pattern is between 60 and $70 \mathrm{~nm}$. This finding is in perfect agreement with results obtained by Kong et al. [28]. Besides the typical stripe pattern, a cellular patter can be found in some areas of electropolished aluminum, especially near pits or dislocations (see Fig. 1c). Some recent works $[28,34]$ claim that the kind of nanostructures obtained during electropolishing depends not only on applied voltage but also on duration of the process. Moreover, the size of nanosized patterns formed on the $\mathrm{Al}$ surface during electropolishing increases with the applied potential [33]. The detailed investigation on this phenomenon is now in progress.

The SEM image of aluminum oxide layer after first anodization is shown in Fig. 2b. The irregular, nanoporous structure with randomly distributed pores of $20-30 \mathrm{~nm}$ in diameter can be easily recognized. A brief comparison of images shown in Fig. 2a,b indicates that the nanopattern created on the aluminum surface by electropolishing is then replicated during the anodic oxide formation. To confirm this, a nanopore/
Fig. 2 SEM images of Al surface after electropolishing (a), aluminum oxide after the first step of anodizing (b), metal surface after oxide removal (c), and porous alumina after second step of anodization (d). The surface area of the samples was $2.25 \mathrm{~cm}^{2}$. Anodization was carried out in $0.3 \mathrm{M}$ oxalic acid at $20^{\circ} \mathrm{C}$ under $45 \mathrm{~V}$. The duration of first and second anodizing step was 1 and 4 h, respectively
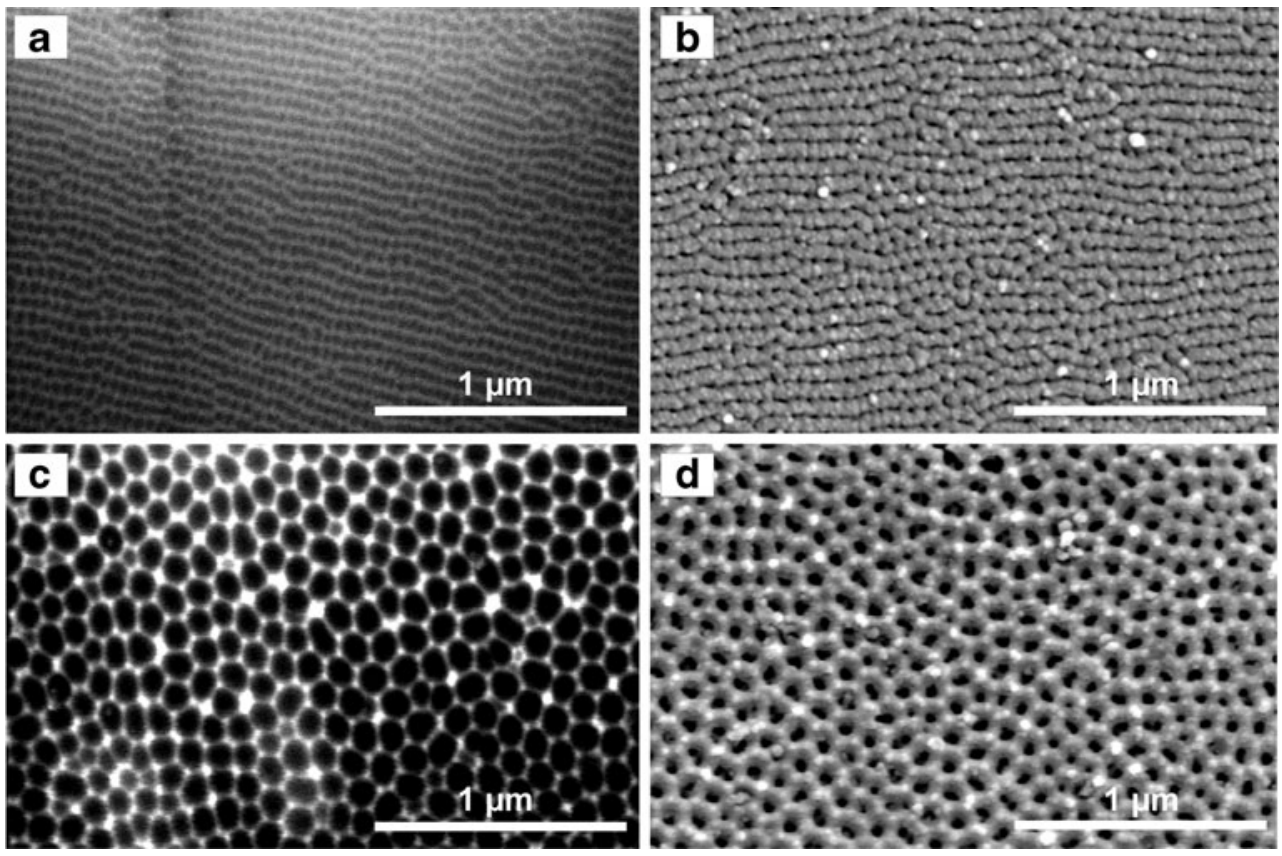
nanostripe spacing was calculated for all samples before and after first anodizing (Fig. 3). The results prove that the morphology of initial nanoporous oxide is strongly determined by the morphology of aluminum substrate used as a starting material. In our experiments, after the first anodizing step, we observed initial growth of pores along the linear nanopattern formed during electropolishing. Below this linear pore structure, the hexagonally arranged pores are visible. This suggest that, according to the mechanism proposed by Jessensky et al. [35], re-arrangement of pores occurs with anodizing time and repulsive forces between the neighboring pores play a crucial role in the porous oxide growth.

According to the point defect model (well describing the pore initiation during anodization) $[36,37]$ and generally accepted the field-assisted dissolution model, the growth of porous oxide occurs at the metal/oxide interface or very close to this interface $[26,38,39]$. During the pore rearrangement process (see below), the size of pores formed near the metal surface increases, while the pore density decreases, mainly due to the merging of adjacent pores. In consequence, the oxide structure found underneath exhibits much larger pore diameters and interpore distances than that initially formed at the beginning of anodization. The thickness of outer layer, with the structure determined by the nanopattern formed during electropolishing, is then reduced during anodizing due to a field-assisted dissolution of $\mathrm{Al}_{2} \mathrm{O}_{3}$ at the oxide/electrolyte interface occurring in the acidic electrolyte. This fact is well illustrated in Fig. $3 \mathrm{~b}$ where beneath the surface, a fragment of an inner oxide layer with much larger pores and pore spacing unveiled during anodizing can be easily seen.

A typical morphology of aluminum surface after oxide removal is shown in Fig. 2c. As can be seen, a dense array of hexagonally arranged concaves is formed during the first anodizing step. These shallow concaves serve as initiation points for the pore formation during second anodizing. An average spacing between these nanoholes was calculated for all anodized samples. It was found that the average distance between concaves, which depends mainly on the applied potential [4], equals to $115 \pm 10 \mathrm{~nm}$ for $45 \mathrm{~V}$ independently of surface area of the sample. The potential of $45 \mathrm{~V}$ was chosen as a potential at which the best hexagonal nanopore arrangement is observed [4].

The SEM image of nanoporous oxide after the second anodization is shown in Fig. 2d. As can be easily seen, pore diameter and interpore distance are larger than those observed just after the first anodizing step; that can be proved by comparison of values from Figs. $3 \mathrm{a}$ and 4a. Moreover, a much better hexagonal arrangement is observed for the oxide structure after the second anodizing step. This is obviously a direct result of the fact that nanoconcaves formed on the $\mathrm{Al}$ surface during the first anodization initiate pore nucleation during the second anodization. For the anodized samples with different surface areas, all structural features of as-obtained porous anodic alumina layers were determined directly from SEM images. Firstly, all nanopores were identified by ImageJ software [21] and such parameters as area and center of mass coordinates were determined for each pore. These raw data were next preceded by the dedicated executable publication $[22,23]$ in order to obtain the average pore diameter $\left(D_{\mathrm{p}}\right)$, interpore distance $\left(D_{\mathrm{c}}\right)$, pore density $(\rho)$, porosity $(\sigma)$, and degree of pore order. The effect of anode surface area on structural features of anodic alumina is shown in Fig. 4. The results indicate that pore diameter increases with increasing surface area of the aluminum sample exposed to the anodizing electrolyte. Moreover, a slight increase in interpore distance can be observed. As a consequence, thinner cell walls are observed for samples with large surface areas (see Fig. 4a).

Due to the lack of regular, hexagonal arrangement of pores, pore density, defined as number of pores per $1 \mathrm{~cm}^{2}$ surface area, and porosity, defined as a ratio of the surface area
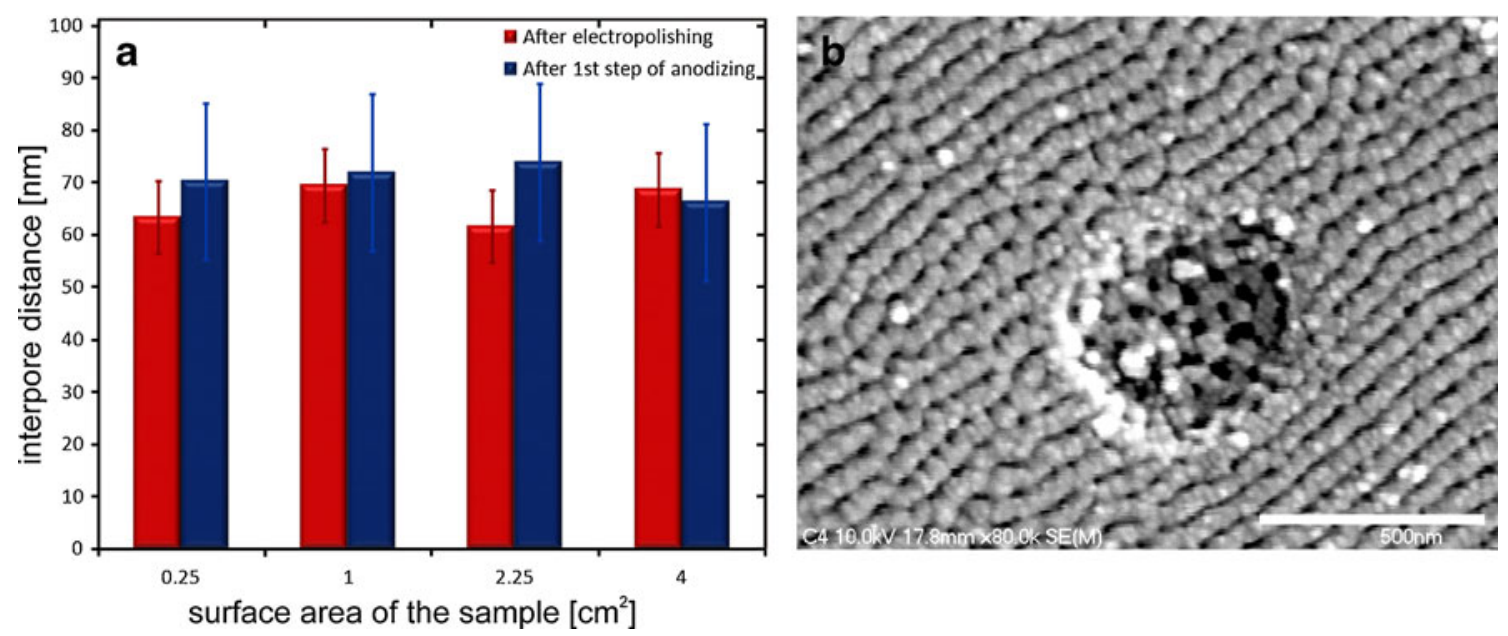

Fig. 3 A comparison between pore to pore distance after electropolishing and after the first step of anodizing (a). SEM image of nanoporous oxide formed at the sample with a surface area of $2.25 \mathrm{~cm}^{2}$ after the first anodizing step (b) 

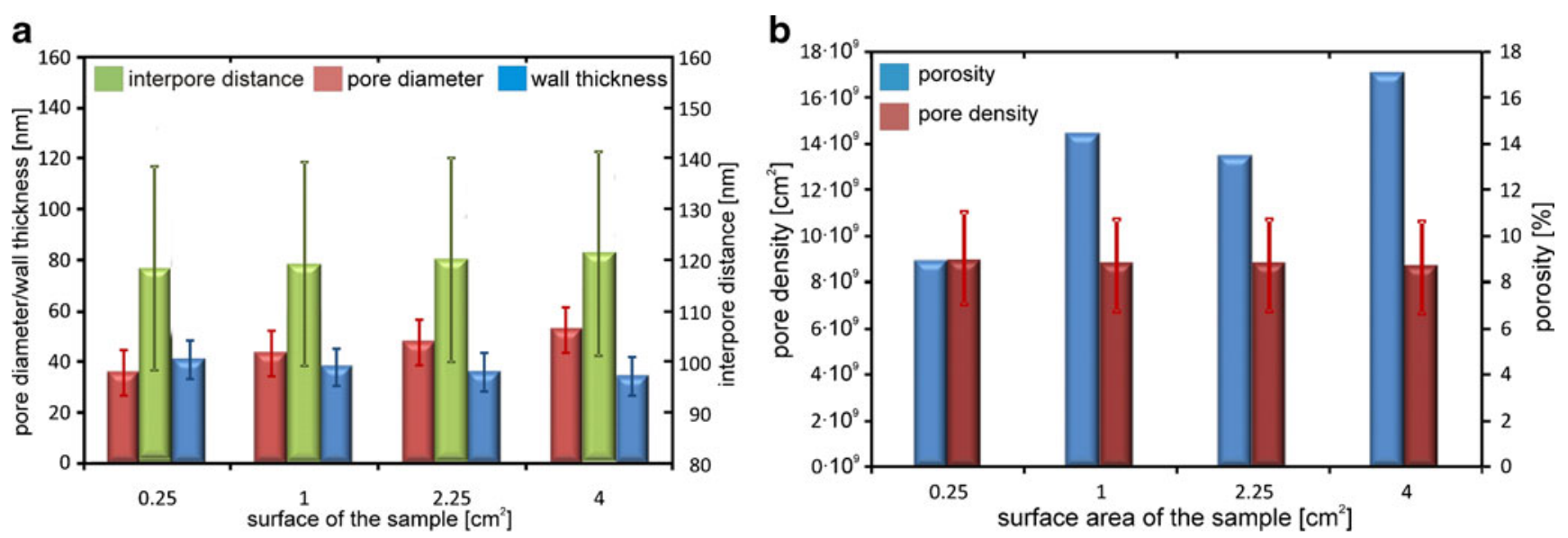

Fig. 4 Values of interpore distance, pore diameter, and wall thickness (a), porosity and pore density (b) for samples with different surface areas after the second step of anodization

occupied by pores to the whole surface area of the sample, were calculated directly from SEM images. The results are shown in Fig. 4b. With increasing surface area of the sample, a slight decrease in pore density was observed; however, this dependence is not statistically significant. It is an obvious consequence of increasing pore spacing. On the other hand, structures with higher porosities were obtained when samples with large surface areas were used for anodization.

A degree of nanopore order was also investigated. The nanopore arrangement for samples with the lowest and highest surface areas was visualized by applying a color coding algorithm proposed by Hillebrand et al. [40]. This approach is based on assigning colors to pores which differ in the relative angular orientation of their basic pattern. As a result, any transition between domains is indicated as a change in the colors of individual pores. Defect maps, known as Delaunay triangulations, were constructed on the basis of pore centers coordinates by using dedicated executable paper powered by GridSpace 2 Virtual Laboratory $[22,23]$ (Fig. 5). The pores not sixfold coordinated by neighboring pores were recognized as defects and marked in black. As can be seen, nanostructures with a quite poor hexagonal arrangement are obtained when a low purity aluminum is used as a starting material and this is in agreement with our previous findings [17]. The percentage of defects, defined as a ratio between the number of defective pores and the number of all pores on the analyzed surface was found to be about $40 \%$ for all studied samples independently of the anode surface area.

Typical dependences between current density and time recorded during anodizations are shown in Fig. 6. In all cases, a rapid decrease in current density is observed during the initial period of anodization as a result of the compact and highresistant oxide layer growth on the $\mathrm{Al}$ surface. After reaching the local minimum, an increase in current density, being a direct consequence of transformation of the continuous oxide layer into porous layer, is observed. Then, the curve shows the desired value which is the evidence of the pore rearrangement process. Finally, the current density does not change significantly with time and a steady-state formation of porous oxide occurs. As can be seen in Fig. 6a, there is a significant
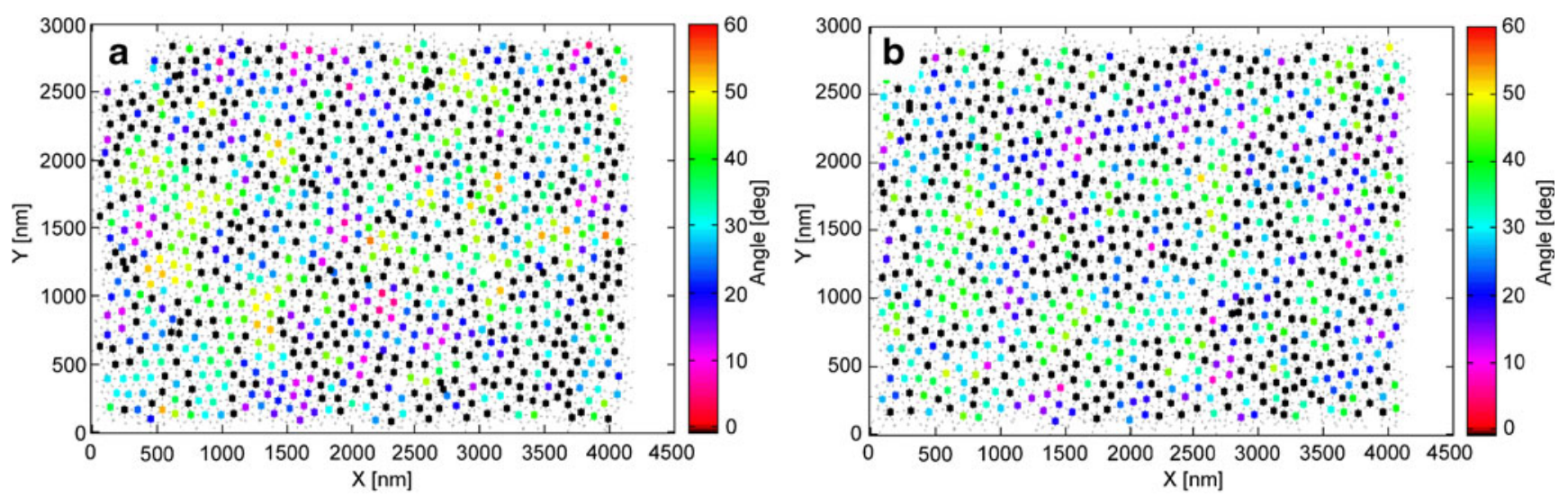

Fig. 5 Maps of pores after applying the color coding algorithm for samples with surface area of $0.25 \mathrm{~cm}^{2}$ (a) and $4 \mathrm{~cm}^{2}$ (b) after the second step of anodization 

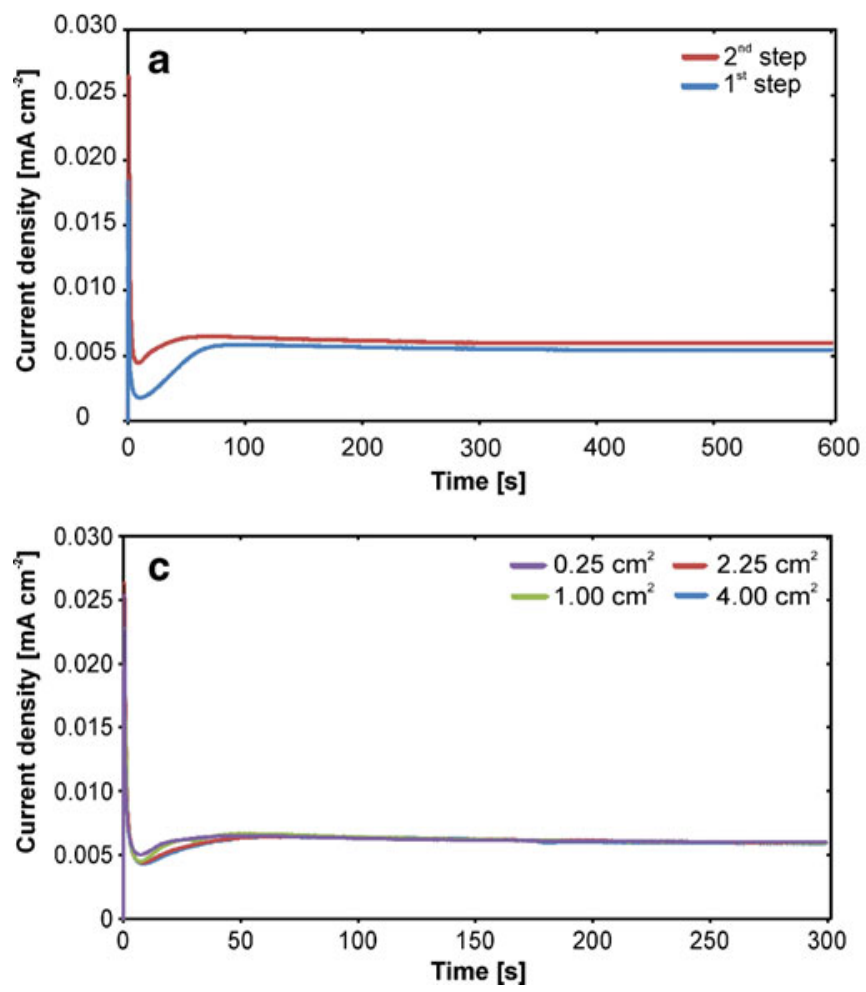

Fig. 6 Current density vs. time curves recorded during the AA1050 alloy anodization. Comparison between the first and second anodizing step (a), curves recorded during the first anodization of samples with different

difference in the current density vs. time dependence for first and second anodizing steps. When the anodic alumina film grows, the current density minimum observed during the first anodization is deeper than in the case when the aluminum surface is pretextured and used for the second anodization. This can be attributed to the fact that at the bottom of each concave, the lowest resistance and highest electric field are observed. Therefore, the pore nucleation occurs much easier on the pretextured surface. In consequence, the lower rate of barrier oxide growth and the more rapid increase in current density are

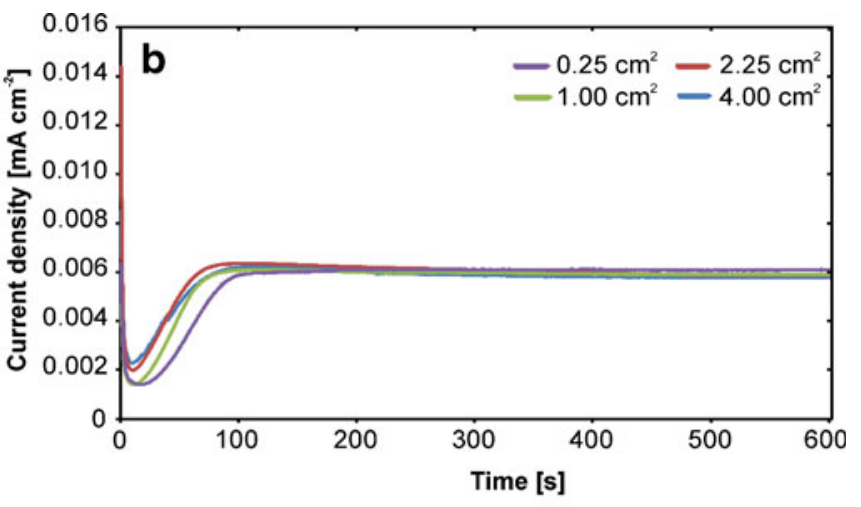

surface areas (b), curves recorded during the second anodization of samples with different surface areas (c)

observed. It should be mentioned that the current-time curve recorded for the AA1050 foil is very similar to those obtained by Li et al. [26] and Wang et al. [41] for high-purity aluminum. For initial $10 \mathrm{~min}$ of first anodizations, current vs. time dependences were recorded for all samples with different surface areas. As can be seen in Fig. 6b, the higher surface area of the anode, the local current density minimum is reached faster and the increase in current density corresponding to the pore rearrangement process is observed earlier. This could be attributed to the fact that the higher surface area of the anode is the
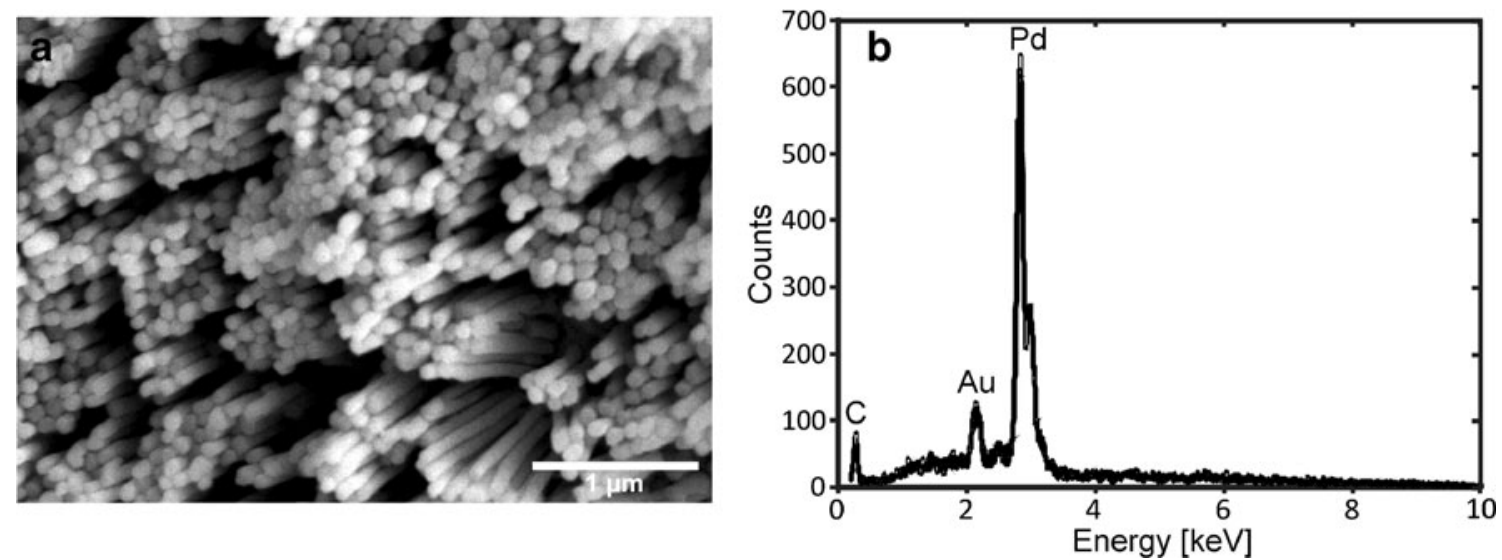

Fig. 7 FE-SEM image of Pd nanowires (a) together with their EDX spectrum (b). The DC electrochemical deposition was performed at $2 \mathrm{~mA} \mathrm{~cm}^{-2}$ for $60 \mathrm{~min}$ 
more defects, like bumps, holes etc., on the metal surface exists. Obviously, the density of defects, i.e., number of defects per surface area unit, is expected to be the same but for large surface areas, mechanical integrity of the barrier oxide layer is more difficult to be maintained and breaking of the continuous dielectric layer can occur earlier. On the other hand, an opposite trend was found when current vs. time curves recorded during the second anodizing step were compared. As can be seen in Fig. $6 c$, the minimum in current density is reached faster for samples with a low surface area. Moreover, not only the time after which the current density minimum is observed but also the depth of this minimum increase with increasing surface area of the anode. It would indicate that the sample with the smallest surface area was pretextured more effectively during the first step of anodization.

The AAO templates with a surface area of $4 \mathrm{~cm}^{2}$ were used as templates for the fabrication of dense array of Pd nanowires. As it was mentioned above, just after the second anodizing step, the remaining aluminum was chemically removed by immersion in the solution of $\mathrm{HCl}+\mathrm{CuSO}_{4}$. Then, the continuous oxide layer at the pore bottoms was etched in a phosphoric acid solution (for details see "Experimental" section) and through-hole AAO templates were obtained. After sputtering of the thin Au layer on AAO, Pd was electrochemically deposited inside the pores of alumina template. A typical SEM image of the Pd nanowire array released from the alumina template together with the EDS analysis is shown in Fig. 7. The dense array of continuous, homogenous in shape, and parallel Pd nanowires with an average diameter of about $90 \mathrm{~nm}$ can be easily recognized. The recorded EDS spectrum confirmed the composition of nanowires; however, the $\mathrm{Au}$ and $\mathrm{C}$ peaks resulting from the thin layer sputtered before electrodeposition on the bottom side of the alumina template and from the conductive carbon paste used during SEM imaging are also noticeable. This kind of Pd electrodes can be a very promising material for many potential applications, e.g., sensors [42, 43] and catalysis [44]; however, some problems with uniform filling of nanopores with metal still should be solved.

\section{Conclusions}

The effect of anode surface area on the nanoporous oxide formation during anodic oxidation of low-purity aluminum was studied in detail. It was confirmed that an ordered nanostripe or nanopore structure is formed on the $\mathrm{Al}$ surface during electrochemical polishing and the wavelength of the created stripe pattern was found to be between 60 and $70 \mathrm{~nm}$. It was revealed that the nanopattern created on the aluminum surface by electropolishing is then replicated during the anodic oxide formation. During the pore rearrangement process, the size of pores formed near the metal surface increases, while the pore density decreases due to the merging of adjacent pores. A comparison of morphology of nanoporous oxide layers formed after the second step of anodizing indicated that the pore diameter, interpore distance, and porosity increase slightly with increasing surface area of the aluminum sample exposed to the anodizing electrolyte. On the other hand, with increasing surface area of the sample, a slight decrease in pore density and cell wall thickness was observed. Current-time behavior was also investigated for all anodized samples. It was found that the higher surface area of the anode during the first step of anodization, the local current density minimum is reached faster and the increase in current density corresponding to the pore rearrangement process is observed earlier. An opposite trend was found when current vs. time curves recorded during the second anodizing step were compared. Finally, we demonstrated a successful fabrication of Pd nanowire arrays ( $\sim 90 \mathrm{~nm}$ in diameter) using nanoporous anodic alumina membranes with relatively large surface areas $\left(4 \mathrm{~cm}^{2}\right)$.

Acknowledgments The research was partially carried out with the equipment purchased thanks to the financial support of the European Regional Development Fund in the framework of the Polish Innovation Economy Operational Program (contract no. POIG.02.01.00-12-023/08). This work was made possible with assistance of the PL-Grid project, contract number: POIG.02.03.00-00-007/08-00, website: www.plgrid.pl. The project is co-funded by the European Regional Development Fund as part of the Innovative Economy program. The SEM imaging was performed in the Laboratory of Field Emission Scanning Electron Microscopy and Microanalysis at the Institute of Geological Sciences, Jagiellonian University, Poland.

Open Access This article is distributed under the terms of the Creative Commons Attribution License which permits any use, distribution, and reproduction in any medium, provided the original author(s) and the source are credited.

\section{References}

1. Zhai T, Yao J (eds) (2013) One-dimensional nanostructures: principles and applications. Wiley, Hoboken, NJ

2. Chen S (ed) (2010) Nanomanufacturing. American Scientific, New York

3. Sulka GD, Zaraska L, Stępniowski WJ (2011) In. Nalwa HS (Ed). Encyclopedia of nanoscience and nanotechnology, vol 11, 2nd ed. American Scientific: California. pp 261-349

4. Sulka GD (2008) In: Eftekhari A (ed) Nanostructured materials in electrochemistry. Wiley, Weinheim, pp 1-116

5. Sulka GD, Stroobants S, Moshchalkov V, Borghs G, Celis JP (2002) J Electrochem Soc 149:D97-D103

6. Sulka GD, Parkoła KG (2006) Thin Solid Films 515:338-345

7. Zaraska L, Sulka GD, Jaskuła M (2011) J Solid State Electrochem $15: 2427-2436$

8. Zaraska L, Stępniowski WJ, Ciepiela E, Sulka GD (2013) Thin Solid Films 534:155-161

9. Aerts T, Dimogerontakis T, De Graeve I, Fransaer J, Tercyn H (2007) Surf Coat Technol 201:7310-7317

10. Montero-Moreno JM, Sarret M, Müller C (2007) Surf Coat Technol 201:6352-6357

11. Montero-Moreno JM, Sarret M, Müller C (2007) J Electrochem Soc 154:C169-C174

12. Yu C, Hu C, Bai A, Yang Y (2007) Surf Coat Technol 201:7259-7265 
13. Bai A, Hu C, Yang Y, Lin C (2008) Electrochim Acta 53:2258-2264

14. Fernández-Romero L, Montero-Moreno JM, Pellicer E, Peiró F, Cornet A, Morante JR, Sarret M, Müller C (2008) Mater Chem Phys 111:542-547

15. Shih T, Wei P, Huang Y (2008) Surf Coat Technol 202:32983305

16. Montero-Moreno JM, Belenguer M, Sarret M, Müller CM (2009) Electrochim Acta 54:2529-2535

17. Zaraska L, Sulka GD, Szeremeta J, Jaskuła M (2010) Electrochim Acta 55:4377-4386

18. Zaraska L, Sulka GD, Jaskuła M (2010) Surf CoatTechnol 205:24322437

19. Michalska-Domańska M, Norek M, Stępniowski WJ, Budner B (2013) Electrochim Acta 105:424-432

20. Horcas I, Fernández R, Gómez-Rodríguez JM, Colchero J, GómezHerrero J, Baro AM (2007) Rev Sci Instrum 78(013705):1-8

21. Image J. National Institute of Mental Health. Bethesda, MD, USA. http://rsb.info.nih.gov/ij

22. Ciepiela E, Zaraska L, Sulka GD (2012) Lect Notes ComputSci 7136:240-251

23. Ciepiela E, Zaraska L, Sulka GD. Implementation of algorithms of quantitative analysis of the grain morphology in self-assembled hexagonal lattices according to Hillebrand method. Executable publication powered by GridSpace2platform, hosted in Academic Computer Centre CYFRONET AGH, http:/gs2.cyfronet.pl/epapers/hillebrand-grains/

24. Nowakowski P, Ciepiela E, Harężlak D, Kocot J, Kasztelnik M, Bartyński T, Meizner J, Dyk G, Malawski M (2011) Proc Comput Sci 4:608-617

25. Shimizu K, Kobayashi K, Skeldon P, Thompson GE, Wood GC (1997) Corros Sci 39:701-718

26. Li F, Zhang L, Metzger RM (1998) Chem Mater 10:2470-2480
27. Guo DZ, Hou SM, Shen ZY, Zhao ZY, Liu WM, Xue ZQ (2002) Chin Phys Lett 19:385-388

28. Kong LB, Huang Y, Guo Y, Li HL (2005) Mater Lett 59:1656-1659

29. Ghorbani M, Nasirpouri F, Irajizad A, Saedi A (2006) Mater Design 27:983-988

30. Rauf A, Mehmood M, Asim Rasheed M, Aslam M (2009) J Solid State Electrochem 13:321-332

31. Sarkar J, Gopal Khan G, Basumallick A (2009) Nanotechnology 20(095604):1-7

32. Huang K, Huang SM, Pu L, Shi Y, Wu ZM, Ji L, Kang JY (2010) Nanoscale Res Lett 5:1977-1981

33. Wang N, Zhang WD, Xu JP, Ma B, Zaho ZZ, Jin QY, Bunte E, Hupkes J, Bochem HP (2010) Chin Phys Lett 27(066801):1-4

34. Yuzhakov VV, Chang SC, Miller AE (1997) Phys Rev B 56:1260812624

35. Jessensky O, Müller F, Gösele U (1998) Appl Phys Lett 72:1173-1175

36. Macdonald DD (2011) Electrochim Acta 56:1761-1772

37. Macdonald DD (1992) J Electrochem Soc 139:3434-3449

38. Thompson GE (1997) Thin Solid Films 297:192-201

39. Zhu XF, Song Y, Liu L, Wang CY, Zheng J, Jia HB, Wang XL (2009) Nanotechnology 20(475303):1-7

40. Hillebrand R, Muller F, Schwirn K, Lee W, Steinhart M (2008) ACS Nano 2:913-920

41. Wang N, Zhang W, Xu J, Ma B, Zhang Z, Jin Q, Bunte E, Hüpkes J, Bochem HP (2010) J Solid State Electrochem 14:1377-1382

42. Im Y, Lee C, Vasquez RP, Bangar MA, Myung NV, Menke EJ, Penner RM, Yun M (2006) Small 2:356-358

43. Wen D, Guo S, Dong S, Wang E (2010) Biosens Bioelectron 26:1056-1061

44. Sun Z, Cheng F, Dai X (2009) J Autom Methods Manag Chem $496281 / 1-5$ 